\title{
Matemáticas y Síndrome de Down.
}

\author{
(Mathematics and Down's Syndrome.)
}

Dolores García López

Universidad de Jaén (España)

Fecha recepción: 11-11-2017

Páginas 119-223

Fecha aceptación: 01-12-2017

\section{Resumen.}

El objetivo principal del presente trabajo es presentar una propuesta didáctica la cual va dirigida a una niña con Síndrome de Down en la que el objetivo principal es afianzar los conocimientos relativos a la comprensión del significado de la posición de un número ya que éste será la base del aprendizaje de conceptos posteriores y fundamentales del currículo matemático. A tal fin, la presente investigación estará estructurada en dos partes. La primera de ellas corresponde al análisis de la base de conocimientos propios de la población que me ocupa, el alumnado con Síndrome de Down. La segunda parte del mismo tendrá como objetivo principal plasmar la propuesta didáctica de matemáticas dirigida a una alumna con Síndrome de Down. Para la elaboración de la siguiente investigación se ha empleado una metodología cualitativa.

Palabas clave: síndrome de Down; competencia matemática

\section{Abstract.}

The principal objective of this investigation is show a didactic proposal what the priority objective is strengthen the knowledges relatives to the understanding of meaning's number since this will be the basis of the learning to futures concepts and paramount concepts of Mathematics' curriculum. For this purpose, the Final Project Degree is structured in two parts. The first one correspond to the analysis of the basis of the own knowledge of the student what I deal, the Down syndrome's students. The second part is aim is reflect the didactic proposal of Mathematic directed to a schoolgirl of the Down syndrome's of Jaén and province. For the project development is exert a qualitative methodology.

Keywords: Down syndrome; mathematical competence 


\section{Introducción.}

El alumnado con Síndrome de Down ha sido y es el alumnado con necesidades educativas especiales más investigado a lo largo de la historia. A pesar de ello, la concepción del mismo no ha sido la misma a lo largo de la historia. Afortunadamente ésta ha ido evolucionando favorablemente a lo largo de la historia...Desde denominarlos con el término "mongolo" hasta hablar de necesidades educativas especiales.

En lo que respecta al plano educativo son múltiples las singularidades que posee el alumnado con Síndrome de Down que infieren en el proceso de enseñanzaaprendizaje las cuales serán el punto de partida de la siguiente programación didáctica. El principal objetivo de toda educación y sobretodo del alumnado con Síndrome de Down es educar para y en la vida, es decir, formar a personas competentes en la sociedad que les rodea. Para alcanzar dicho objetivo será de vital importancia apostar por una educación de calidad y sobretodo, normalizada e inclusiva. Una educación en la que el alumnado sea el protagonista partiendo de las necesidades que presente el mismo.

\section{Justificación.}

Para poder participar activamente en la sociedad que nos rodea actualmente es necesario ser competente en el plano matemático ya que vivimos en un mundo cuantificado en el que los números están presentes en la inmensa mayoría de labores diarias. Por tanto, podemos decir que la realización de la siguiente investigación surge de la necesidad de educar para y en la vida.

No obstante, las escasas investigaciones realizadas a lo largo de la historia en el campo de la didáctica de las matemáticas con el alumnado con Síndrome de Down postulan las grandes dificultades que presenta la población que nos ocupa ante el aprendizaje de conceptos matemáticos.

\section{1.- El Síndrome de Down.}

Cuando hablamos de Síndrome de Down aludimos a un síndrome cuyo origen es genético al hallarse en él una alteración en el número de cromosomas, habiendo un cromosoma de más, 47 en vez de 46 . En consecuencia, este alumnado presenta cierto grado de discapacidad intelectual adjunta a otras características físicas y una mayor incidencia en algunas enfermidades como son problemas cardíacos, entre otros. (Huete, 2016) (Rodríguez y Olmo, 2010)

Los primeros documentos que aportan datos relativos al síndrome que nos ocupa corresponden a Esquirol y Seguin. El primero de ellos, llevó a cabo un estudio en su primer libro en el que describía a un conjunto que poseía un tipo de de discapacidad intelectual ligado a una serie de características similares a las que presenta el alumnado con Síndrome de Down. (Cammarata-Scalisi, Da Silva, CammarataScalisi, Adalgisa, 2010) 
No obstante, no fue hasta el siglo XIX, cuando se conoció la primera descripción científica, la cual fue aludida a John Langdon Haydon Down en la que se hace referencia a un tipo de deficiencia intelectual ligada a otras características similares a las del Síndrome de Down. En consecuencia, la llamada Trisomía 21 procedió a denominarse Síndrome de Down, en honor al honorable autor. (Down, 1866. Citado en Garrido, Muñoz, García y Salado, 2015) (Chávez, 2014)

Una fecha a destacar en el marco histórico del Síndrome de Down es el año 1959, ya que gracias a Lejeune, Turpin y Gautier el síndrome que nos ocupa pasó a contemplarse desde el plano genético al encontrar una anomalía en el número de cromosomas. (Pérez, 2014) (Ortega, 2006)

Tal y como he postulado anteriormente, el origen del Síndrome de Down es genético debido a una alteración en el número del cromosomas. Dentro del mismo plano genético podemos encontrar tres variantes del Síndrome de Down las cuales expondré a continuación de mayor a menor incidencia.

- Trisomía Libre: en la que la alteración genética está presente en la totalidad celular del individuo debido a que en la fase de meiosis no se separó correctamente un cromosoma.

- Translocación: en la que el origen de la anomalía genética se encuentra en "una rotura a nivel estructural, dando como resultado un fragmento cromosómico libre" (Pérez, 2014, p. 2358)

- Mosaicismo: en la que la alteración genética no se encuentra en la totalidad celular del individuo .En consecuencia, un individuo mosaico puede presentar características propias del Síndrome de Down y otras que no. El porcentaje de células trisómicas en un individuo es, una vez más, muy variable. (Ortega, 2006) (Basile, 2017) (Rodríguez y Olmo, 2010)

Actualmente, no se conocen la "causas" que producen la trisomía o indicios de la existencia de la misma. Debido a ello hablaré de factores de riesgo que puedan estar relacionados con la incidencia del mismo. Entre los factores de riesgo a destacar encontramos que el porcentaje de nacidos con el síndrome es mayor en aquellos casos en los que la edad de la madre supera los 35 años.

En lo que respecta al ámbito educativo son múltiples las singularidades que posee el alumnado con Síndrome de Down que infieren en el proceso de enseñanzaaprendizaje las cuales serán el punto de partida de la siguiente programación didáctica.

De las principales singularidades he destacado las siguientes, en líneas generales: discapacidad intelectual acompañada de un claro retraso del lenguaje. Así mismo, el proceso de la información se caracteriza por ser lento y dificultoso. Por otro lado, encontramos déficits en los procesos de atención y memoria, en concreto la auditiva y de trabajo por lo que los resultados incrementarán positivamente si se emplea material visual. 
No obstante, en la población que nos ocupa todo no son adversidades, el alumnado que me ocupa destaca en lo referido a lo social; en consecuencia, la enseñanza de las matemáticas será más efectiva si se parte de experiencias...en definitiva, si se apuesta por un aprendizaje funcional y significativo.

\section{2.-Matemáticas y Síndrome de Down.}

A lo largo de la historia los estudios llevados a cabo son escasos en comparación a la literatura concerniente al estudio de habilidades referidas a la lectura en esta población. Afortunadamente, esta realidad ha cambiado ya que me consta de un mayor número de estudios. Grosso modo, podemos observar dos grandes grupos:

En un primer lugar, encontramos un grupo en los que se analizan las "barreras" que presenta el alumnado con Síndrome de Down en el área matemática. En segundo lugar, encontramos un gran número de investigaciones en las que el principal objeto de estudio es el análisis del momento de aparición de los principios de conteo y el modo en el que éstos lo adquieren.

Finalmente, he querido destacar un tercer grupo de estudios relativamente recientes en los que se estudian y evidencian los beneficios del empleo del material multimedia en la didáctica de las matemáticas con el alumnado que nos ocupa como es el caso de la investigación llevada a cabo por Doña Juana María Ortega Tudela el cual he tomado como uno de mis referentes a la hora de la elaboración de mi material didáctico.

A pesar del crecimiento del número de investigaciones, actualmente, no existe una metodología específica por lo que he realizado un análisis de los estudios encontrados el cual me ha permitido extraer una serie de directrices las cuales han sido el punto de partida de mi material didáctico:

- Diversidad: Cada niño y niña con Síndrome de Down es diferente. Por tanto, el punto de partida será el estudio y análisis de las habilidades que posee el del alumnado.

- Déficits en lo relativo a la memoria y en la atención como ya se comentó anteriormente por lo que la información a presentar ha de ser realmente atractiva y motivante.

- El progreso disminuye cuando la información a mostrar es presentada por el canal auditivo. En consecuencia, la metodología y estrategias a utilizar han de ser visuales y atractivas.

- Dificultades en tareas que conlleven abstracción y generalización por lo que el profesional ha de secuenciar cuidadosamente los pasos que conlleven a la adquisición de una habilidad numérica.

- Los contenidos a trabajar han de estar directamente relacionados con experiencias diarias con el fin de trasladar lo aprendido al quehacer diario. 


\section{3.-El alumnado con Síndrome de Down desde el plano legislativo.}

Cuando hablamos del alumnado con SD desde el plano legislativo hemos de hacer referencia al concepto específico de necesidades educativas especiales tal y como queda establecido en el Título II "Equidad en la Educación" de la Ley Orgánica de Educación. Un alumno y/o alumna presenta necesidades educativas especiales cuando en un período concreto de su escolarización o también, a lo largo de toda ella, precise de determinados apoyos y atenciones educativas de carácter específico.

He de destacar que cuando se alude a la escolarización de cualquier alumno y/o alumna la legislación establece que ésta ha de estar regida por los principios de normalización e inclusión. En consecuencia, para conseguir esos principios se ha de partir de la identificación temprana de las citadas necesidades educativas especiales

\section{4.-Propuesta didáctica.}

\section{1.-Descripción del caso.}

Antes de comenzar a detallar la siguiente procederé a presentar la alumna a la que va dirigida el vigente material didáctico. A la hora de referirme a ella emplearé el nombre ficticio de María. María tiene 13 años, cursa quinto de Educación Primaria en un centro ordinario aunque su nivel curricular corresponde a primero de Educación Primaria. Por otro lado, ésta asiste a clases de apoyo semanales y logopedia en uma asociación, a la cual asisto en la modalidad de voluntaria.

Para la elaboración del presente perfil he recogido los datos obtenidos en la prueba inicial realizada al comienzo del segundo trimestre del vigente curso escolar en la clase de apoyo a la cual asisto en la modalidad de voluntaria en dicha asociación.

En líneas generales, María es capaz de reconocer y escribir números comprendidos entre el 0 y el 99 presentado dificultad en la grafía de algunos números como el 78. Dichas dificultades se basan en la inversión de las cifras, escritura de un número cuyo sonido sea similar a otro. De este último error, se evidencia lo postulado anteriormente en en lo que respecta a la dificultad de interiorización de la información a través del canal auditivo. En lo que respecta al plano del cálculo, María es capaz de realizar adiciones y sustracciones sin llevadas apoyándose en el conteo de dedos. En cuarto y último lugar, la alumna que nos ocupa no ha asimilado el concepto de decena y por tanto no es capaz de reconocer el valor posicional de las cifras de un número de dos cifras y sus respectivas equivalencias.

\section{2.-Objetivos y contenidos.}

Como objetivo general encontramos: Comprender el Sistema de Numeración Decimal y su respectivo significado así como aplicar el mismo a la vida cotidiana, haciendo de él un saber funcional. En lo relativo a los objetivos específicos encontramos: 
1. Enunciar el Sistema de Numeración Decimal desde el 0 al 99.

2. Relacionar el valor de un número con su correspondiente posición en un número.

3. Asumir y apreciar el valor del saber Matemático en lo que respecta al quehacer diario.

4. Tender a participar positivamente en las actividades propuestas, así como hacer un buen uso de las tecnologías de la información y comunicación en el proceso de enseñanza-aprendizaje.

Los contenidos a trabajar en la presente unidad didáctica corresponden al significado del valor posicional del número. En consecuencia, éstos se encuentran recogidos en el bloque 2. Números principalmente.

\section{3.-Actividades}

La unidad didáctica diseñada tiene como título " $\mathrm{jJugamos}$ y aprendemos a contar con Ninal". Ésta ha sido estructurada en cuatro tareas que parten de experiencias significativas para María.

- Primera tarea: La fiesta de cumpleaños de Nina: La primera tarea tiene como fin introducir el concepto de decena y su respectivo valor. Para ello se ha estructurado ésta en dos actividades. Una primera en la que María ha de colocar en cada bolsita una decena de caramelos que en este caso serán canicas. Una vez colocada cada decena de canicas se le preguntará a la alumna por el número de decenas que se ha conseguido. $Y$ en segundo lugar, una actividad lúdica en la que María ha de contar diversos ítems y posteriormente ha de descomponer el número en decenas y unidades cada número.

- Segunda tarea: Ayudamos a ordenar el cuarto de Nina:La segunda tarea que se ha llevado a cabo tiene como fin introducir y poner en práctica las equivalencias del valor posicional de un número y ha sido estructurada en dos actividades. En la primera de ellas María ha de agrupar y contar diversos ítems. Para ello, se le ha indicado a la alumna de que la manera más rápida y fácil de hacerlo será formando decenas. La segunda de ellas se ha basado en un juego en el que la alumna ha de formar parejas con las fichas expuestas en el pupitre.

- Tercera tarea: Imagina ser frutera: Ésta tiene como fin reforzar lo aprendido a lo largo de las sesiones anteriores y presentar apoyo en aquellas dificultades presentadas. Para ello, he diseñado una actividad lúdica que simula una frutería en la que ha de ayudar a atender a la clientela dándole la cantidad de fruta indicada. En dichos pedidos se verán reflejadas cantidades descompuestas en unidades y decenas.

- Cuarta tarea: Un sábado en casa de la abuela: La cuarta y última tarea tiene como fin evaluar lo aprendido en la unidad didáctica. Para ello he diseñado dos actividades lúdicas como son un bingo y una última que se 
basará en un juego en el que la alumna ha de ayudar a Nina y su abuela a comprar las cantidades (expresadas tanto descompuestas y cardinales).

\section{4.-Metodología.}

La misma ha estado regida, principalmente, por el perfil de la alumna a la que va dirigida el presente material didáctico. Así mismo, la siguiente metodología está basada en las conclusiones extraídas del análisis de las líneas anteriores del presente Trabajo de Fin de Grado. Los principios sobre los que se ha enmarcado los estructuraré en dos secciones: principios generales y específicos.

\section{Principios generales:}

En los principios generales encontramos aquellos que han de ser el referente en toda metodología. Dichos principios se encuentran recogido em el Título II "Equidad en la Educación", concretamente en el artículo 74, la educación de cualquier alumno y alumna se ha de regir por los principios de normalización e individualización.

\section{Principios específicos:}

Los principios específicos a presentar están basados principalmente en el perfil de la alumna y en las conclusiones extraídas del análisis de las investigaciones no médicas llevadas a cabo en el campo que nos ocupa.

- Individual: la presente unidad didáctica es individual ya que va enfocada a una alumna solamente y además, es individual porque parte de las habilidades que posee la alumna.

- Activa: es de suma importancia seguir una metodología en la que se mantenga activa la atención de la alumna y así compensar el déficit de atención.

- $\quad$ Cualitativa: porque el punto de partida son las habilidades de la alumna.

- Flexiva: tiene como epicentro a la alumna por lo que ha sido posible adecuar en todo momento el proceso de enseñanza-aprendizaje a las necesidades que manifieste en el trascurso de la unidad didáctica.

- Visual: es visual ya que, como anteriormente se ha citado, el alumnado con Síndrome de Down obtiene mejores resultados ofreciendo la información por el canal visual que por el canal auditivo.

- Motivadora: es motivadora debido a que el alumnado con Síndrome de Down y en concreto la alumna a la que va dirigida la presente muestra un déficit de atención por lo cual es de suma importancia lograr mantener su atención.

- $\quad$ Participativa: es participativa porque se precisa de la participación de la alumna en todo momento, siendo ella la principal protagonista del proceso de enseñanza-aprendizaje.

- Lúdica: es lúdica porque el proceso de enseñanza-aprendizaje parte de juegos manipulativos y visuales.

- Integradora: es integradora porque se pretende formar a la alumna en todas sus posibilidades de cara al futuro evitando así inculcar un conocimiento de forma aislada. 
- Funcional: se ha seguido una metodología que parte de experiencias cercanas a su quehacer diario fomentando de este modo la extrapolación de los contenidos a enseñar.

- Significativa: es significativa porque el referente en el proceso de enseñanza-aprendizaje es lo ya conocido y experimentado.

\section{5.-Propuestas de mejora.}

Para la identificación de propuestas de mejora es necesaria la evaluación de lo trabajado a lo largo de la presente programación. Dicha evaluación ha sido carácter continuo ya que ha tomado como punto de partida la evaluación inicial. Seguidamente se ha procedido con un seguimiento del progreso de María a través de la observación directa del mismo. Finalmente, como pueden ver en la diapositiva se ha empleado una rúbrica en la que se han evaluado las habilidades de la alumna.

\section{Conclusión.}

La puesta en práctica de la unidad didáctica me ha permitido confirmar que el progreso del alumnado con Síndrome de Down aumentaba a través de metodologías visuales y atractivas ya que la alumna pudo mantener la atención en períodos de tiempo de mayor prolongación. Así mismo, he podido evidenciar la gran dificultad que presenta esta población ante tareas que conlleven mecanismos de abstracción y generalización mostrando, de este modo, más problemas en la actividad concerniente a la Tarea 3: Imagina ser frutera.

La evaluación de la programación expuesta me ha permitido observar como la alumna, a diferencia de la evaluación inicial, asociaba el concepto de decena a su respectivo valor e identificaba la misma en conjuntos mayores. No obstante, María presenta a veces, no de forma repetitiva, dificultades en el valor posicional de algunos números, en concreto, aquéllos superiores al 59 y en los números cuyas cifras sean iguales, como por ejemplo 77 u 88 . Por lo que se deberá seguir reforzando el valor posicional del número para así afianzar dicho contenido.

Finalmente, he de decir que una educación que apuesta por una metodología individualizada, normalizada e inclusiva favorece el desarrollo de la enseñanza del alumnado con necesidades educativas especiales, en concreto, el alumnado con Síndrome de Down.

\section{Bibliografía.}

Baroody, A. J. (1988). El pensamiento matemático de los niños. Un marco evolutivo para maestros de preescolar, ciclo inicial y educación especial. Madrid, España: Aprendizaje Visor y MEC. 
Baroody, A.J. (1988).Number-comparasion learning by children classified as Mentally Retarded. American Journal on Mental Retardation. Vol. 92, No 5, pp. 461471.

Basile, H. (2017).Retraso mental y genética Síndrome de Down. Alcmeon, Revista Argentina de Clínica Neuropsiquiátrica, 15(1), pp. 9- 23.

Bird, G; Buckley, S. (2010). Desarrollo de habilidades numéricas y motoras para alumnos con Síndrome de Down y acceso a las tecnologías de la información. Madrid: CEPE, S.L.

Bird, G; Buckley, S. (2010). En Bird, G \& Buckley, S (2010). Habilidades numéricas en alumnos con síndrome de Down. Una visión de conjunto. Desarrollo de habilidades numéricas y motoras para alumnos con Síndrome de Down y acceso a las tecnologías de la información. Madrid: CEPE, S.L.

Brown, A.L. y DeLoache, J.S. (1978). Skills, plans and self-regulation. En R.S. Siegler (Ed.). Children's thinking: What develops? Hillsdale, NJ. England.

Bruno, A., Noda, A. (2014) Comprensión del sistema de numeración decimal de una alumna con síndrome de Down. En M. T. González, M. Codes, D. Arnau y T. Ortega (Eds.), Investigación en Educación Matemática XVIII (pp. 177-186). Salamanca: SEIEM.

Buckley, S., Bird, G. (2001). Number skills for individuals with Down Syndrome. An Overview. Down Syndrome Issues and Information. Southsea, Hampshire: The Down Educational Trust.

Byrne, A.; Buckley, S., MacDonald, J. y Bird, G. (1995). Investigating the literacy, language and memory skills of children with Down's syndrome. Down's Syndrome: Research and Practice, 3, pp. 53-8.

Cammarata-Scalisi, F., Da Silva, G., Cammarata-Scalisi, G., Adalgisa Sifuentes C. (2010). Historia del síndrome de Down. Un recuento lleno de protagonistas. Can Pediatr, 34 (3), pp.157-159.

Carr, J. (1988). Six weeks to twenty-one years old: A longitudinal study of children with Down syndrome and their families. J Child Psychol Psyquiatry 29(4), pp.407-431.

Caycho, L; Gunn, P.; Siegal, M. (1991). Counting by children with Down Syndrome. American Journal on Mental Retardation, 95(5), pp. 575-583.

Cornwell, A.C. (1974). Development of language, abstraction and numerical concept formation in Down's syndrome Children. American Journal of Mental Deficiency, 79 (2), pp.179-190. 
Costa, A.B.; Picharillo, A.D.M.; Elias, N.C. (2017).Avaliação de habilidades matemáticas em crianças com síndrome de Down e com desenvolvimento típico. Ciênc. Educ., Bauru, 23(1), pp. 255-272.

Flórez, J. (1999). Bases neurológicas del aprendizaje. Siglo Cero. 30(3), pp. 9-27.

Flórez, J. (1999). Síndrome de Down. Presentación General. Siglo Cero. 30(4), pp.515.

Flórez, J. (2016). Síndrome de Down en perspectiva 2016. Revista Síndrome de Down, 33(1). pp. 16-23.

Flórez, J.; Troncoso, M. V. (1991). Síndrome de Down y Educación. Masson S.A. and Fundación Síndrome de Down de Cantabria, 33, pp. 16-23.

Garrido, F; Muñoz, M; García, P y Salado, M (2015). Efectos beneficiosos del karate sobre la propiocepción y la coordinación en jóvenes con Síndrome de Down. Revista médica internacional Síndrome de Down,19(1), pp. 14-17.

Gelman, R. (1982). Accessing one-to-one correspondence: Still another paper about conservation. British Journal or Psychology,73(2), pp. 209-220.

Manassero, G. (2016). Guía de práctica clínica del Síndrome Down. Rev. Fac. Med. Hum.,16(1),pp. 37-45.

MECD (2015). Orden ECD/65/2.015. BOE. № 25.

MECD (2006). LEY ORGÁNICA 2/2006, de 3 de mayo, de Educación. BOE de 4 de mayo de 2006.Título II "Equidad en la educación". MECD (2014).

Real Decreto 126/2014, de 28 de febrero, BOE. № 52.

Noda, A, Bruno, A. (2017).Assessment of the Knowledge of the Decimal Number System Exhibited by Students with Down Syndrome. Qualitative Research in Education, 6(1), pp.5685.

Noda. A y Bruno. A. (2010).Operaciones básicas en alumnos con Síndrome de Down. PNA, 4(4), pp. 143-159.

Ortega, J. M. (2006). Nuevas tecnologías y Aprendizaje Matemático en niños con Síndrome de Down. II Premio en investigación no médica en Síndrome de Down. Madrid, España: FEISD y Obra Social Caja de Madrid. 